\title{
Object Flow Definition for Refined Activity Diagrams
}

\author{
Stefan Jurack ${ }^{1}$, Leen Lambers ${ }^{2}$, Katharina Mehner ${ }^{3}$, Gabriele Taentzer ${ }^{1}$, \\ and Gerd Wierse ${ }^{1}$ \\ ${ }^{1}$ Philipps-Universität Marburg, Germany \\ \{sjurack, taentzer, gwierse\}@mathematik.uni-marburg.de \\ ${ }^{2}$ Technische Universität Berlin, Germany \\ leen@cs.tu-berlin.de \\ ${ }^{3}$ Siemens, Corporate Technology, Germany \\ katharina.mehner@siemens.com
}

\begin{abstract}
Activity diagrams are a well-known means to model the control flow of system behavior. Their expressiveness can be enhanced by using their object flow notation. In addition, we refine activities by pairs of pre- and post-conditions formulated by interrelated object diagrams. To define a clear semantics for refined activity diagrams with object flow, we use a graph transformation approach. Control flow is formalized by sets of transformation rule sequences, while object flow is described by partial dependencies between transformation rules. This approach is illustrated by a simple service-based on-line university calendar.
\end{abstract}

\section{Introduction}

UML2 activity diagrams are a well-known means to model the control flow of system behavior. Their expressiveness can be enhanced by using their object flow notation. Currently, it is an open problem how to formalize coherent object flow for activity diagrams. In this paper we aim at providing a precise semantics for refined activity diagrams with coherent object flow. We use graph transformation as semantic domain, since it supports the integration of structural and behavioral aspects and provides different analysis facilities.

In [1], sufficient criteria for the consistency of refined activity diagrams were provided, where interrelated object diagrams are used to specify pre- and post conditions of single activities. All conditions refer to a domain class model. This refinement serves as a basis for consistency analysis. The refinement of activities by pre- and post-conditions was first introduced in [2] to analyze inconsistencies between individual activities refining use cases. Pre- and post conditions are formalized as graph transformation rules. Mehner et.al. extend the consistency analysis in [3] where also the control flow is taken into account. In [4], a similar approach for consistent integration of life sequence charts (LSCs) with graph transformation, applied to service composition modeling, was developed. The formalization based on graph transformation is used to analyze rule sequences. In addition, data flow is modeled textually by name equality for input and output variables.

In this paper, we extend refined activity diagrams by object flow. We introduce partial rule dependencies to formalize the semantics of object flow. Based on the consistency 
notion of refined activity diagrams in [1], we define consistency-related properties of refined activity diagrams with object flow.

We illustrate our approach with an example from model-driven development of a service-based web university calendar. In particular the behavior modeling of individual services still lacks advanced support for precise modeling and subsequent consistency analysis. Activity diagrams are an adequate means for modeling individual services, and the use of object flow and pre-/post-conditions can define service behavior more precisely.

This paper is organized as follows. Section 2 introduces the syntax and semantics of refined activity diagrams with object flow informally. Section 3 introduces algebraic graph transformations and the new notion of partial rule dependency. Section 4 presents the semantics and consistency notion of refined activity diagrams and extends it for object flow. Sections 5 and 6 contain related work and concluding remarks.

\section{Introduction to Refined Activity Diagrams with Object Flow}

This section introduces refined activity diagrams with object flow and illustrates this modeling approach by a small example for a service-based web university calendar. In this example, we model services by activity diagrams with object flow where each activity is refined by pre- and post-conditions, and guards are refined by patterns.

\subsection{Domain Model}

Our example application manages course parts that are lectures, laboratories, and exercises where a lecture may offer a laboratory and an exercise. Each course part is held by a lecturer and can be located in a room. An appropriate class diagram is presented in Fig. 1. From an abstract class Object 1, three classes are derived: Room, Lecturer and CoursePart. The latter is abstract and is specialized by three further classes: Laboratory, Exercise and Lecture. Day and time information for course parts are realized by enumerations Day and Time.

\subsection{Activity Diagrams with Object Flow}

We use UML2 activity diagrams with object flow [5] to model services of the university calendar. Three services, AddLecture, AddExercise, and AddLaboratory, are shown exemplarily in Fig. 2.

Web applications usually contain a number of services. A service provides a clearly defined logical unit of functionality based on data entities. While a basic service might be realized by one activity only, more complex services might contain a number of different activities. Defining services by the means of hierarchical activity diagrams opens up the possibility to call services from other ones. The usage of other services is depicted by placing a complex activity as representation of the used service into the control flow. The invocation of a complex activity is indicated by placing a rakestyle symbol within the activity node. Our example service AddLecture uses two other

\footnotetext{
${ }^{1}$ Italic class names in diagrams indicate abstract classes.
} 


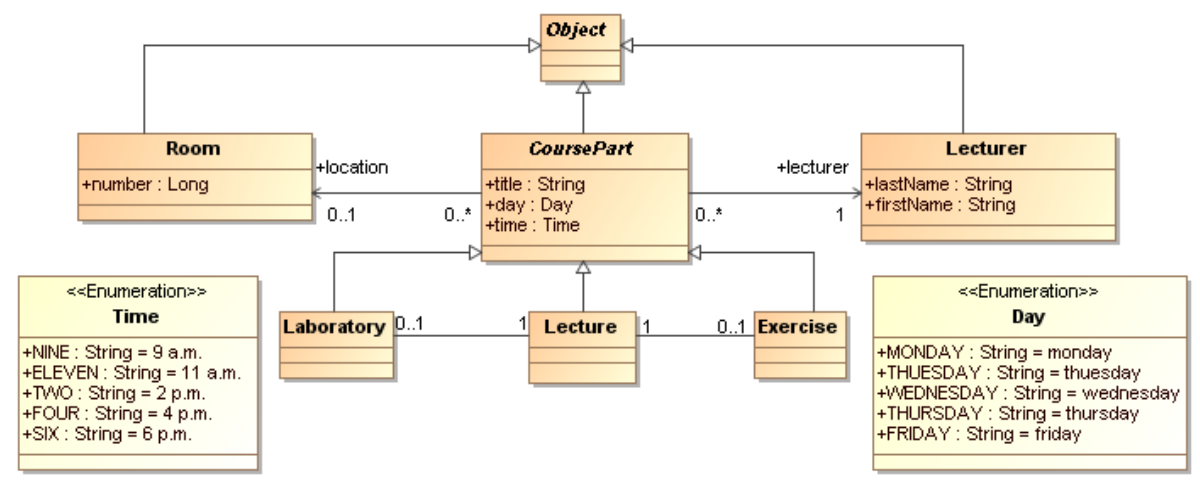

Fig. 1. Domain Class Diagram

services. Accordingly, the complex activities modeling used services AddLaboratory and AddExercise are refined by corresponding activity diagrams (cf. Section 4).

UML2 provides several object flow notations. The preference for a notation depends on different aspects, e.g. the amount of information, potential ambiguities, and the equality of control and object flow. For example, if object and control flow overlap, related objects may be depicted next to transitions as shown above activity SetRoom in Fig. 2, Otherwise an object node with separate object flow edges has to be used as shown for lecture $l$. However, it is desirable to keep the object flow description as simple as possible without leaving out important information. Each object may be named and its identity is expressed by equal names within an activity diagram. E.g. in activity diagram AddLecture both lecturer nodes named $l 2$ depict the same object. Please note that in our approach, an object may flow along multiple outgoing edges i.e. object flows, whereas in UML2 one object serves one object flow exclusively.

Objects passed from outside to an activity diagram can be drawn on the diagram boundary in order to show parameters flowing into certain activities. Objects passed out of the diagram itself, may be depicted as boundary objects as well. Consider Fig. 2 , Objects of types Lecturer and Room are passed to the activity diagram AddLecture, while a newly created object of type Lecture is passed out of this diagram.

In Fig. 2, service AddLecture uses two other services AddLaboratory and AddExercise. Once a lecture has been created and its attributes have been set, a related laboratory or exercise might be created additionally. At first, a new lecture is created in activity CreateLecture, its attributes are set and it is linked to lecturer $l 1$. If, moreover, room $r l$ is not null, activity SetRoom is used to link this room $r l$ to the lecture newly created. If a lecturer is given for a laboratory, the complex activity AddLaboratory is used to add a laboratory to the lecture. Therefore, AddLecture has to pass the newly created lecture $l$, lecturer 12 , and room $r 2$ to the activity. In diagram AddLaboratory a new laboratory is created by the first activity CreateLaboratory. In the same step, this laboratory is linked to lecture $l$ and to lecturer $l 2$. Furthermore, the laboratory's attributes are set. In the next activity, the laboratory's location is set to room $r 2$, provided that $r 2$ is given. If a lecturer for a related exercise is given, AddExercise is used by AddLecture analogously. 
AddLecture(in String lecTitle, in Day lecDay, in Time lecTime, in String labTitle, in Day labDay, in Time labTime, in String exTitle, in Day exDay, in Time exTime, in Lecturer I1, in Room r1, in Lecturer I2, in Room r2, in Lecturer I3, in Room r3, out Lecture I)

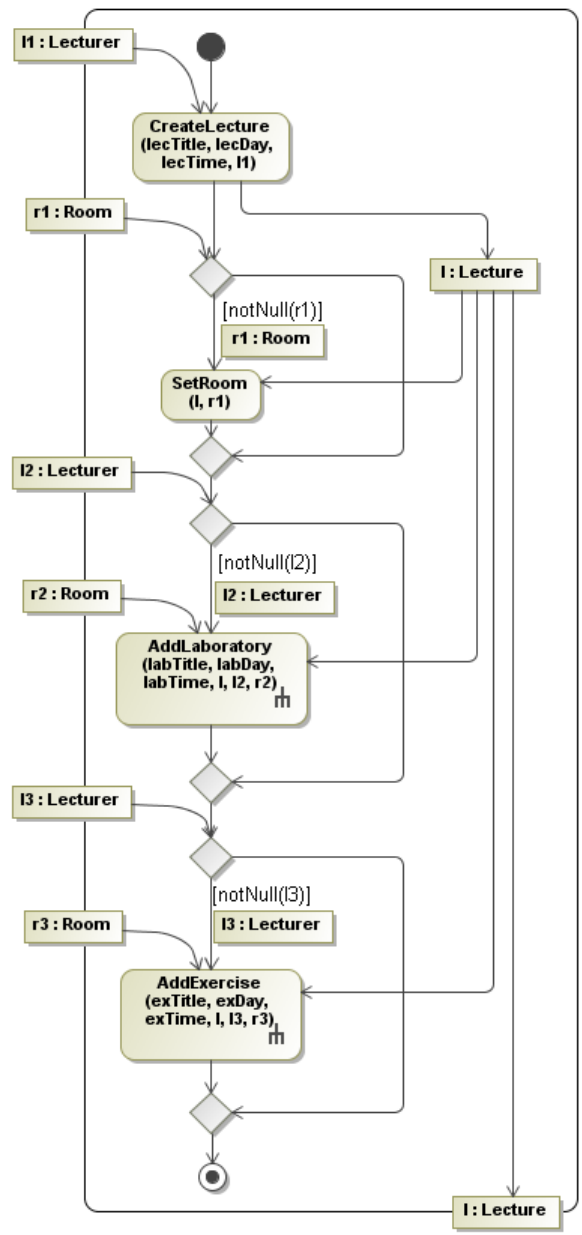

AddLaboratory(in String labTitle, in Day labDay, in Time labTime, in Lecture I, in Lecturer I2, in Room r2)

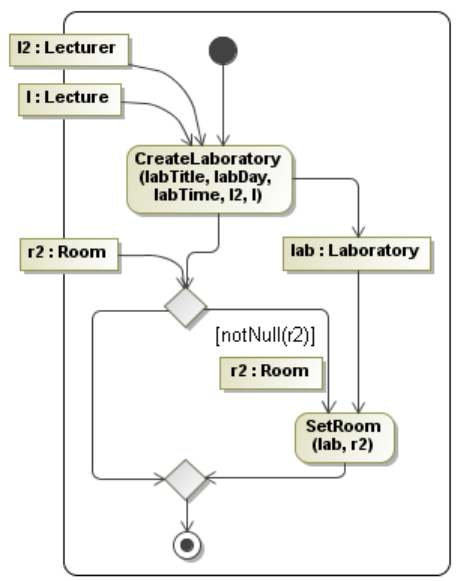

AddExercise(in String exTitle, in Day exDay, in Time exTime, in Lecture I, in Lecturer I3, in Room r3)

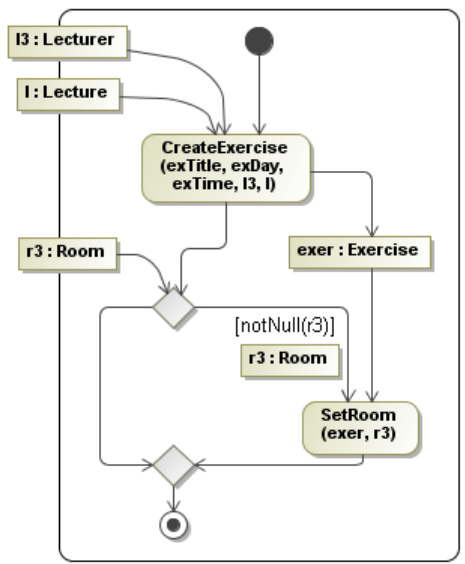

Fig. 2. Activity Diagrams of Services AddLecture and AddLaboratory

Since our activity diagrams model services, we equip each of them with a name and a comma-separated list of parameters. The semantics follow the programming concept of parameter passing between operations, i.e. an activity diagram models an operation consisting of a signature and a body. The signature of an activity diagram consists of its name and a list of attribute and object parameters. While object parameters have a type occurring in the domain model, attribute parameters have primitive types in most cases. This signature is an extension of UML2 made by our approach. Please note that all attributes and boundary objects used within the activity diagram are arguments which correspond to the signature. In addition, each parameter declaration has to be enriched with keyword in, out, or inout. This qualification defines the object flow direction. E.g. 
lecturer $l 1$ has to be passed to diagram AddLecture and is therefore marked in. Vice versa, the newly created lecture $l$ is passed out of the diagram and is therefore marked by out. Parameter objects marked by inout are both input and output objects.

\subsection{Refined Activities}

Activities are used to model specific changes of the current system snapshot i.e. object structure. We propose to refine activities by pre- and post-conditions specifying snapshots before and after the activity respectively. We refine activities separately by pairs of object diagrams which are typed over the domain model. Figure 3 shows object diagrams refining activities of our example (cf. Fig. 2) where pre-conditions are depicted on the left and post-conditions on the right. Objects and links with equal names on both sides express identity and preservation. Objects and links occurring on the left-hand side only will be deleted, while objects and links occurring in the right-hand side only will be created. Conditions on non-existence of patterns are depicted in red dashed outline.

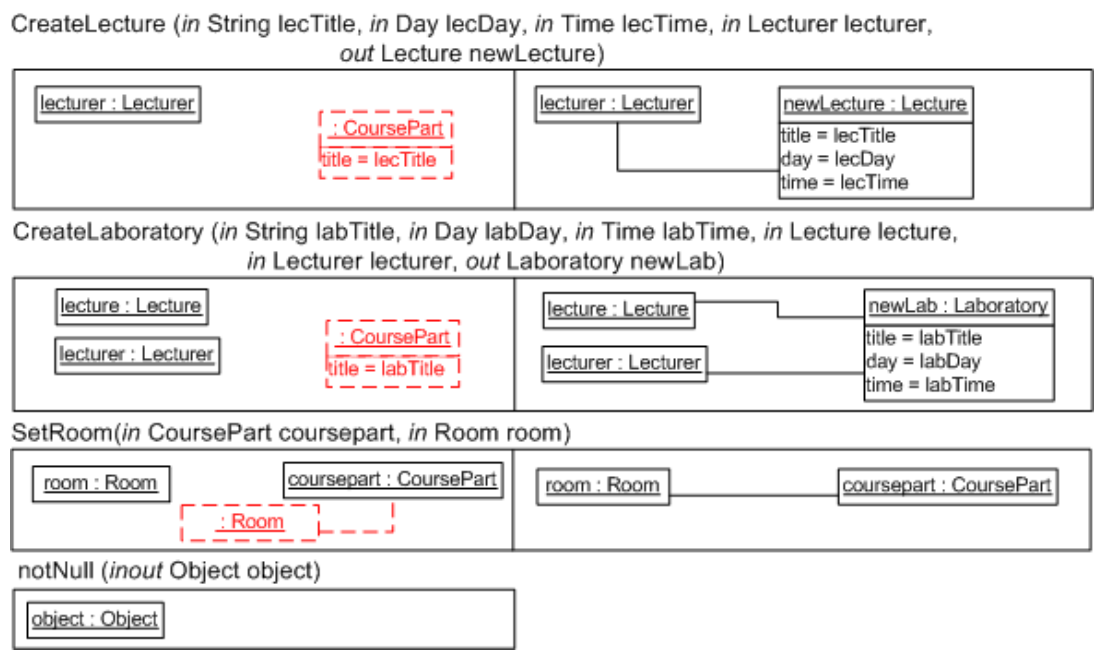

Fig. 3. Refined Activities by Pre- and Post-Conditions

Each pair of conditions exhibits a signature according to the inscription of its refined activity, i.e. it consists of a name (the activity name) and a list of typed parameters qualified with keyword in, out or inout. Parameters can be distinguished into object and attribute parameters, analogously to their usage in activity diagrams. While the former ones are matched to objects, the latter ones are used as attribute values. Keyword in requires the occurrence of the related object (if object parameter) on the left-hand side. The object may be used in a read, edit, or delete operation. Keyword out declares a returned object and requires its presence on the right-hand side. It may be used for a create or select operation. Inout declares an object to be given and returned as well, thus requires the given object on both sides which explicitly guarantees its non-deletion. Attribute parameters must be input parameters. If occurring in pre-conditions, attribute 
parameter values restrict the matching of objects, occurring in post-conditions they are used to assign attribute values. Object parameter types must be respected by condition checking, i.e. by pattern matchings. Parameters may be matched, if they are matched with equally typed or sub-typed values only. Analogously, this must hold for attribute types. Note that arrays and collection-like types are not supported by our approach yet.

The first pair of conditions in Fig. 3 refines activity CreateLecture. The pre-condition requires the existence of a lecturer in the current system snapshot, otherwise the activity cannot be applied. Also, it requires the non-existence of a CoursePart instance (which could be of concrete type Lecture, Exercise, or Laboratory) with a title equal to given attribute parameter lecTitle. If both conditions hold, the activity is applicable and creates a Lecture instance associated with the given Lecturer instance and the lecture is returned. The refinement of activity CreateLaboratory shown as second pair in Fig. 3 is quite similarly, but it requires two given objects to exist and the creation of an object of type Laboratory. Since the conditions of CreateExercise are analogous to those of CreateLaboratory, they are left out. The refinement of activity SetRoom is shown as third pair. It requires two object parameters, one instance of type Room and one of type CoursePart, and it forbids the CoursePart instance to have a room already. No object but a link between the given course part and the new room is created here. Please note, that CoursePart is an abstract type. Thus instances of its concrete sub-classes can be used here only The last condition in Fig. 3 refines guard notNull. Since guards do not perform model-changing transformations but rather check for existence in the system snapshot, we just define a guard pattern here. Note that we disallow non-existence conditions in guard patterns. Else-guards are predefined by negated guard patterns i.e. it is checked for non-existence of the corresponding guard pattern.

\section{Formalization by Graph Transformation}

The UML variant presented in the previous section can be equipped with a graph transformation semantics. We start with presenting the theory of graph transformation as in [6] and extend it by new concepts. While class diagrams are formalized by type graphs, activities with pre- and post-conditions are mapped to graph rules. The object flow is formalized by a new concept called partial rule dependencies. This semantics definition serves as a basis for validating the consistency of refined activity diagrams with object flow.

\subsection{Graphs and Graph Transformation}

Graphs are often used as abstract representation of visual models, e.g. UML models. When formalizing object-oriented models, graphs occur at two levels: the type level (defined by a meta-model) and the instance level. This idea is described by the concept of typed attributed graphs, where a fixed type graph $T G$ serves as an abstract representation of the meta-model (without constraints). Node types can be structured by an inheritance hierarchy and may be abstract in the sense that they cannot be instantiated. Multiplicities and other annotations have to be expressed by additional graph constraints. Attribute types are formally described by data type algebras. Instances of 
the type graph are object graphs equipped with a structure-preserving mapping to the type graph. Attribute values are given by a concrete data algebra.

Graph transformation is the rule-based modification of graphs. A rule is defined by $p=(L \stackrel{l}{\longleftarrow} K \stackrel{r}{\longrightarrow} R, I, O, N A C s)$ where $L$ is the left-hand side (LHS) of the rule representing the pre-condition and $R$ is the right-hand side (RHS) describing the postcondition. $l$ and $r$ are two injective graph morphisms, i.e. functions on nodes and edges which are structure and type-preserving. They specify a partial mapping $r \circ l^{-1}$ from $L$ to $R . L \backslash l(K)$ defines the graph part that is to be deleted, and $R \backslash r(K)$ defines the graph part to be created. The types of newly created nodes have to be non-abstract. Elements in $K$ are mapped in a type preserving way. All graphs of a rule are attributed by the same algebra being a term algebra with variables. Some of these variables are considered to be rule parameters. Input parameters can be nodes or variables, thus $I=I_{N} \cup I_{V}$, whereas output parameters can be nodes only, i.e. $O=O_{N}$ with $I \subseteq L$ and $O \subseteq R$.

NACs is a set of negative application conditions, each defined by an injective graph morphism $n: L \rightarrow N$ where $N \backslash n(L)$ defines a forbidden graph part. $n$ allows to refine node types, i.e. a node of a more abstract type is allowed to be mapped to a node with a finer type according to the inheritance hierarchy.

Example 1 (Example rules). Figure 3 shows example graph rules. Each pre-condition forms an LHS with one negative application condition and each post-condition describes an RHS. Identifiers given by names indicate the mapping between left- and right-hand sides. The solid parts of a pre-condition indicate the LHS $L$, while the dashed ones prohibit a certain graph part and represent $N \backslash n(L)$ of the NAC. Input and output parameters are listed on top of each pair of conditions, formally in the head of each rule.

A graph transformation step $G \stackrel{p, m}{\Longrightarrow} H$ between two instance graphs $G$ and $H$ is defined by first finding a match $m: L \rightarrow G$ of the left-hand side $L$ of rule $p$ into the current instance graph $G$ such that $m$ is an injective type-refining graph morphism. Match $m$ has to fulfill the dangling condition, i.e. nodes may be deleted only, if all adjacent edges are mentioned in the LHS. Moreover, each NAC has to be fulfilled, i.e. $m$ satisfies a $N A C$, if for each $n \in N A C s$ there does not exist an injective typerefining morphism $o: N \rightarrow G$ such that $o \circ n=m$. Input parameters are instantiated by concrete values being nodes of the instance graph and data type values. Thus, parameter instantiation provides a partial match.

In the second step, graph $H$ is constructed by a double-pushout construction (see [6]). Roughly spoken, the construction is performed in two passes: (1) build a graph $D$ which contains all those elements of $G$ not deleted; (2) construct $H$ as a union of $D$ and all elements of $R$ to be created. To focus on the preserved part of a graph transformation step, we define a partial graph morphism track : $G \rightarrow H$ by track $=g^{-1} \circ h$. Graph dom(track) is the subgraph of $G$ where track is defined, i.e. the domain of track. (See also [7] for a first definition of track morphism.) Morphisms $g: D \rightarrow G$ and $h: D \rightarrow H$ are constructed by a double-pushout as shown below. Morphism $g^{-1}$ is always well-defined, since $l$ is injective and the pushout construction preserves injectivity, thus $g$ is also injective. Furthermore, a so-called co-match $m^{\prime}: R \rightarrow H$ is defined by the double-pushout construction. Output parameters point to a certain part 
of this co-match. Output parameters are useful for pointing to specific nodes which can be used in further transformation steps then.

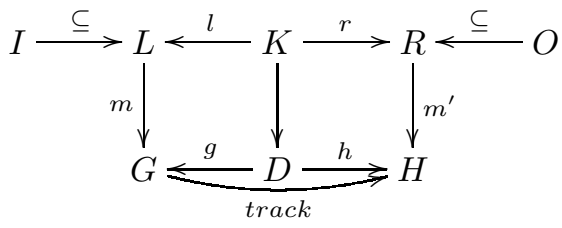

A graph transformation (sequence) $t=G_{0} \stackrel{p_{1}, m_{1}}{\Longrightarrow} G_{1} \ldots G_{n-1} \stackrel{p_{n}, m_{n}}{\Longrightarrow} G_{n}$ consists of zero or more graph transformation steps. Track morphism track $k_{0, n}$ of sequence $t$ is simply the composition of track morphisms $\operatorname{track}_{n-1, n} \circ \ldots \circ$ track $_{0,1}$ of its steps. For $n=0$, track $_{0,0}=i d_{G_{0}}$. A set of graph rules $P$, together with a type graph $T G$, is called a graph transformation system (GTS) $G T S=(T G, P)$. A GTS may show two kinds of non-determinism: Given a graph, (1) several rules can be applicable, and (2) for each rule several matches can exist. There are techniques to restrict both kinds of choices. The choice of rules can be restricted by the definition of control flow while the choice of matches can be restricted by passing partial matches The tool AGG (Attributed Graph Grammar System) [8] can be used to specify and analyze graph transformation systems.

\subsection{Partial Rule Dependencies}

To restrict the choice of matches for rules, we introduce the concept of partial rule dependencies which may relate output parameter nodes of one rule to input parameter nodes of a (not necessarily direct) subsequent rule in a given rule sequence 2 . We say that rule sequences are dependency-compatible, if the transitive closure of all dependencies between each two rules is well-defined.

Definition 1 (partial and joint rule dependencies). Given a GTS (T,P) and a rule sequence $s: p_{1}, \ldots, p_{n}$ with $p_{1}, \ldots, p_{n} \in P$. A partial rule dependency between rules $p_{i}$ and $p_{j}$ with $1 \leq i<j \leq n$ is defined by an injective partial morphism $d_{i j}: O_{i_{N}} \rightarrow I_{j_{N}}$ from output parameter nodes of $p_{i}$ to input parameter nodes of $p_{j}$. If $d_{i j}$ is the empty morphism, no rule dependency is defined. For each pair of rules $p_{i}$ and $p_{j}$ in $s$, its closure $_{i j}$ is defined as follows: (1) $d_{i j}$ belongs to closure ${ }_{i j}$ and (2) for all $d_{i k}, d_{k j}$, and rules $p_{k}$ with $i<k<j$ add $d_{k j} \circ r_{k} \circ l_{k_{\mid I_{k}}}^{-1} \circ d_{i k}$ to closure $e_{i j}$.

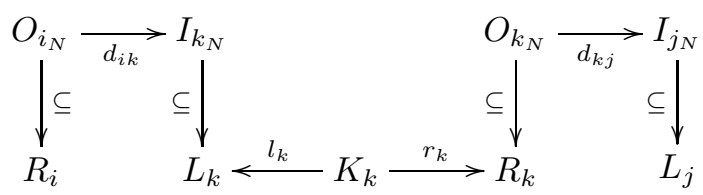

Rule sequence $s$ is dependency-compatible, if for all closures closure ${ }_{i j}$ the following holds: (1) For all $d \in$ closure $_{i j}:$ type $(x)$ has to be finer or coarser than type $(d(x))$

\footnotetext{
${ }^{2}$ Note that rule sequences differ from transformation sequences in not providing graphs to which rules are applied.
} 
for all $x \in O_{i_{N}}$ wrt. the type inheritance relation defined by type graph $T$. (2) Each two dependencies $d$ and $d^{\prime}$ in closure ${ }_{i j}$ are weakly commutative, i.e. $d(x)=d^{\prime}(x)$ for all $x \in \operatorname{dom}(d) \cap \operatorname{dom}\left(d^{\prime}\right)$.

If rule sequence $s$ is dependency-compatible, we can define a joint dependency of a closure. Given closure $i_{i j}$ we define the joint dependency dep $p_{i j}: O_{i_{N}} \rightarrow I_{j_{N}}$ as follows: (1) $\operatorname{dom}\left(\operatorname{dep}_{i j}\right)=\bigcup_{d \in \text { closure }_{i j}} \operatorname{dom}(d)$ and (2) $\operatorname{dep}_{i j}(y)=d(y)$ if $y \in \operatorname{dom}(d)$ for $d \in$ closure $_{i j}$

Example 2 (partial rule dependencies). Considering the rules in Fig. 3, we compose rule sequence $s=$ CreateLecture, SetRoom, CreateLaboratory, SetRoom. As first step, we define partial rule dependencies taking input and output parameters into account: $d_{12}($ newLecture $)=$ coursepart $, d_{23}=d_{34}=d_{24}=d_{14}=\emptyset, d_{13}$ (newLecture $)=$ lecture. All dependencies are type-compatible, since either the types of mapped nodes are equal or in hierarchy, e.g. type(newLecture) $=$ Lecture is finer than type $\left(d_{12}(\right.$ newLecture $\left.)\right)=$ type (coursepart $)=$ Coursepart (see Fig. 1). None of the closures contains more than one non-empty partial dependency. Thus, partial rule dependencies are not really composed from each other in this example, e.g. dep $p_{13}=$ $d_{13}$.

If cour separt were an inout parameter of rule SetRoom, closure , $_{13}$ could look more interesting: With $d_{23}$ (cour separt) $=$ lecture we would have closure cl3 $_{13}=\left\{d_{13}, d_{23}\right.$ 。 $\left.r_{2} \circ l_{2}^{-1} \circ d_{12}\right\}$ with $\operatorname{dep}_{13}($ newLecture $)=$ lecture.

If we enlarged the rule sequence by rule CreateLaboratory and defined $d_{34}$ (newLab) $=$ coursepart as well as $d_{45}$ (coursepart) $=$ lecture, then $d_{e} p_{35}$ would have to map newLab) to lecture which would not be type-compatible.

Definition 2 (application of dependency-compatible rule sequences). A dependency -compatible rule sequence $s: p_{1}, \ldots, p_{n}$ is applicable to some graph $G_{0}$, if there is a graph transformation sequence $G_{0} \stackrel{p_{1}, m_{1}}{\Longrightarrow} G_{1} \ldots G_{n-1} \stackrel{p_{n}, m_{n}}{\Longrightarrow} G_{n}$ such that $m_{j} \circ$ dep $p_{i j}$ and track $_{i, j-1} \circ m_{i}^{\prime}\left(O_{i_{N}}\right)$ are weakly commutative, with track $k_{i, j-1}$ being the track morphism from $G_{i}$ to $G_{j-1}$ and $m_{i}^{\prime}$ being the co-match of rule $p_{i}$ for $1 \leq i<j \leq n$.

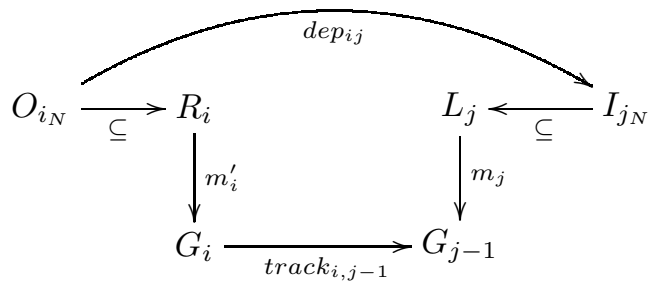

Partial rule dependencies are defined independently of causal dependencies. Causal dependencies between rules can be analyzed by the critical pair analysis (CPA) [6]. The only kind of causal dependencies we are interested in here are produce/usedependencies where the application of one rule produces an element needed by the match of a second rule. If two rules are not causally dependent on each other, the corresponding joint dependency which is defined explicitly must not introduce any produce/use-dependency. If some partial dependency is defined, it has to correspond with at least one produce/use dependency. 


\section{Object Flow: Semantics Definition and Properties}

In this section, we first specify well-structured refined activity diagrams, refine their activities by graph rules and their guards by graph patterns, and define their semantics and consistency based on graph transformation. Thereafter, this approach is extended to refined activity diagrams with object flow.

From now on, we assume that an activity diagram does not contain any complex activities and that each complex activity has been flattened before, i.e. it has been replaced by its refining activity diagram. During this potentially recursive process, each object which goes in to or comes out from a complex activity is glued with the corresponding boundary object of the refining activity diagram, i.e. the boundary and boundary objects disappear.

\subsection{Refined Activity Diagrams}

As in [91], we restrict our considerations to well-structured activity diagrams. The building blocks are simple activities, sequences, fork-joins, decision-merge structures, and loops only.

Definition 3 (well-structured activity diagram). A well-structured activity diagram $A$ consists of a start activity s, an activity block $B$, and an end activity e such that there is a transition between $s$ and $B$ and another one between $B$ and $e$. An activity block is defined as follows:

- Empty: An empty activity block is not depicted.

- Simple: A simple activity is an activity block.

- Sequence: A sequence of two activity blocks $A$ and $B$ connected by a transition from $A$ to $B$ form an activity block.

- Decision/Merge: A decision activity which is followed by two guarded transitions leading to one activity block each and where each block is followed by a transition both heading to a common merge activity form an activity block. One transition is explicitly guarded, called the if-guard, while the other transition carries a predefined guard "else" which equals the negated if-guard.

- Loop: A decision activity is followed by a guarded transition. This guard is called loop-guard. The transition leads to an activity block with an outgoing transition to the same decision activity as above. Considering this decision activity again, its incoming transition from outside becomes the incoming transition of the new block. Its outgoing transition to outside becomes the outgoing transition of the new block. This transition is guarded by "else". The whole construct forms an activity block.

- Fork/Join: A fork activity followed by two branches with one activity block each followed by a join activity form an activity block.

To be able to define object flow to be coherent with control flow we define a control flow relation as prerequisite. Because of potential loops it is not a partial order.

Definition 4 (control flow relation). The control flow relation $C F R_{A}$ of an activity diagram A contains pairs ( $x, y)$ where $x$, $y$ are activities such that the following holds: 
- Pair $(x, y) \in C F R_{A}$, if $x$ is directly connected via a transition with $y$.

- If $(a, b) \in C F R_{A}$ and $(b, c) \in C F R_{A}$, then also $(a, c) \in C F R_{A}$.

An if- or loop-guard is equipped with a graph pattern which describes an existence condition on graphs. A guard pattern can be interpreted as identical rule (i.e. a rule where the left and the right-hand sides are equal). Guard pattern $g$ is fulfilled by a graph $G$, if its corresponding rule $p_{g}$ is applicable to $G$. After rule $p_{g}$ has been performed, the guarded alternative is executed. Otherwise, rule $\bar{p}_{g}$ which formalizes "else" for given guard $g$, is applicable to $G$ and the second alternative is performed.

Definition 5 (guard pattern, guard rule and negated guard rule). A guard pattern $g$ is defined by a typed graph being attributed over a term algebra with variables. Its guard rule $p_{g}$ is defined by $\left(g \stackrel{i d_{g}}{\longleftarrow} g \stackrel{i d_{g}}{\longrightarrow} g, I, O, \emptyset\right)$. Its negated guard rule $\bar{p}_{g}$ is defined by $(\emptyset \stackrel{\emptyset}{\longleftarrow} \emptyset \stackrel{\emptyset}{\longrightarrow} \emptyset, \emptyset, \emptyset,\{n: \emptyset \rightarrow g\})$.

Lemma 1. Given a guard pattern $g$ and a graph $G$. Rule $p_{g}$ is applicable to $G$, iff rule $\bar{p}_{g}$ is non-applicable to $G$.

Proof. See [10].

Definition 6 (refined activity diagram). A refined activity diagram $R A$ is a wellstructured activity diagram such that each simple activity occurring in $R A$ is equipped with a graph transformation rule. Each if- or loop-guard occurring in $R A$ is equipped with a guard pattern. We also say that an activity is refined by a transformation rule where decision activities are refined by guard rules deduced from guard patterns which refine guards.

Definition 7 (semantics of refined activity diagrams). Given an activity block $B$ of a refined activity diagram $R A$, its corresponding set of rule sequences $S_{B}$ is defined as follows.

- If $B$ is empty, $S_{B}=\emptyset$.

- If $B$ consists of a simple activity a refined by rule $p_{a}, S_{B}=\left\{p_{a}\right\}$.

- If $B$ is a sequence of $X$ and $Y, S_{B}:=S_{X}$ seq $S_{Y}=\left\{s_{x} s_{y} \mid s_{x} \in S_{X} \wedge s_{y} \in S_{Y}\right\}$

- If $B$ is a decision block on $X$ and $Y$ with guard pattern $g$ refining its if-guard, $S_{B}=\left(\left\{p_{g}\right\}\right.$ seq $\left.S_{X}\right) \cup\left(\left\{\bar{p}_{g}\right\}\right.$ seq $\left.S_{Y}\right)$

- If $B$ is a loop block on $X$ with guard pattern $g$ refining its loop-guard, $S_{B}:=$ $\operatorname{loop}\left(g, S_{X}\right)=\bigcup_{i \in I} S_{X}^{i}$ where $S_{X}^{0}=\left\{\bar{p}_{g}\right\}, S_{X}^{1}=\left\{p_{g}\right\}$ seq $S_{X}$ seq $\left\{\bar{p}_{g}\right\}$, $S_{X}^{2}=\left\{p_{g}\right\}$ seq $S_{X}$ seq $S_{X}^{1}$ and $S_{X}^{i}=\left\{p_{g}\right\}$ seq $S_{X}$ seq $S_{X}^{i-1}$ for $i>2$. $S_{B}(n)=S_{X}^{n}$ denotes the semantics of loop block $B$ with exactly $n$ loop executions.

- If $B$ is a fork block on $X$ and $Y, S_{B}:=S_{X}\left\|S_{Y}=\bigcup s_{x}\right\| s_{y}$ with $s_{x} \in S_{X} \wedge s_{y} \in$ $S_{Y}$ where $s_{x}\left\|\lambda=\left\{s_{x}\right\}, \lambda\right\| s_{y}=\left\{s_{y}\right\}$, and $p_{x} s_{x}^{\prime} \| p_{y} s_{y}^{\prime}=\left\{p_{x}\right\} \operatorname{seq}\left(s_{x}^{\prime} \| p_{y} s_{y}^{\prime}\right) \cup$ $\left\{p_{y}\right\} \operatorname{seq}\left(p_{x} s_{x}^{\prime} \| s_{y}^{\prime}\right)$.

The semantics $\operatorname{Sem}(R A)$ of a refined activity diagram $R A$ consisting of a start activity $s$, an activity block $B$, and an end activity $e$ is defined as the set of rule sequences $S_{B}$ generated by the main activity block $B$. If $R A$ contains $k$ guarded loops, $\operatorname{Sem}_{n_{1}, \ldots, n_{k}}(R A) \subseteq \operatorname{Sem}(R A)$ denotes a restricted semantics where the semantics of each guarded loop $B_{j} \in A$ for $1 \leq j \leq k$ is $S_{B_{j}}\left(n_{j}\right)$. 
Now, we are ready to check the control flow consistency of activity diagrams. To do so, we consider snapshots of the system, i.e. object models which are formalized as graphs by mapping objects to graph nodes and object links to graph edges. In the following definitions for consistency-related properties, we directly use graphs as abstract syntax representation of object models.

Activity diagrams are consistent, if there is a set $\mathcal{S}$ of model graphs such that each rule sequence in the diagram semantics is applicable to some of these graphs. If the diagram contains guarded loops, we use the restricted semantics for diagrams (as defined above) which checks for each guarded loop, if a predefined number of loop executions is feasible. $\mathcal{S}$ is without junk, if each of its model graphs represents a potential snapshot of the system to which an activity sequence in $A$ can be applied.

Definition 8 (completeness). A set $\mathcal{S}$ of graphs is complete wrt. to a refined activity diagram $R A$, if for all rule sequences $s$ in $\operatorname{Sem}(R A)$ there is a graph $G$ in $\mathcal{S}$ such that $s$ is applicable to $G$. If $R A$ contains $k$ guarded loops, a set $\mathcal{S}$ of graphs is quasi-complete wrt. to $R A$, if for all rule sequences $s$ in $\operatorname{Sem}_{n_{1}, \ldots, n_{k}}(R A)$ there is a graph $G$ in $\mathcal{S}$ such that $s$ is applicable to $G$. Set $\mathcal{S}$ is without junk, if for each graph in $\mathcal{S}$ at least one applicable rule sequence in $\operatorname{Sem}(R A)$ (resp. $\operatorname{Sem}_{n_{1}, \ldots, n_{k}}(R A)$ ) exists.

Definition 9 (consistent activity diagram (without object flow)). A refined activity diagram $R A$ is consistent, if there is a set $\mathcal{S}$ of graphs which is complete wrt. $R A$. If $R A$ contains $k$ guarded loops, $R A$ is quasi-consistent, if there is a set $\mathcal{S}$ of graphs which is quasi-complete wrt. $R A$.

\subsection{Refined Activity Diagrams with Object Flow}

In the following, we define refined activity diagrams by partial rule dependencies which formalize object flows and enrich its semantics.

Definition 10 (well-structured activity diagram with coherent object flow). $A$ wellstructured activity diagram $A_{O F}=(A, O b j, O F R, I, O)$ with coherent object flow is a well-structured activity diagram $A$ (as given in Def. 3) equipped with a set of object nodes $O b j$, an object flow relation $O F R$ for $A$ and $O b j$, input parameter set $I$, and output parameter set $O$, defined as follows:

- Input parameters can be object nodes or values, i.e. $I=I_{N} \cup I_{V}$ with $I_{N} \subseteq$ Obj. Output parameters may only be object nodes only, i.e. $O=O_{N}$ with $O \subseteq O b j$.

- Object flow relation $O F R$ contains triples $(x, o, y)$ where $x$ and $y$ are simple or decision activities and $o \in O b j$. In addition, there is a special tag null not used as activity name which is used to define object flow from and to parameter objects, i.e. triples (null, o, y) and $(x, o, n u l l)$ can also be in $O F R$ where $o \in I_{N}$ or o $\in O_{N}$, resp. For each object o in $I_{N}$ (resp. in $O_{N}$ ), there is a triple (null,o,y) (resp. $(x, o, n u l l))$ in $O F R$. For each other object $o \in O b j$, there has to be a triple $(x, o, y) \in O F R$.

- OFR is coherent with control flow relation $C F R_{A}$ of $A$ (see Def. 4), i.e. for all $(x, o, y) \in O F R$ with $x, y \neq$ null there is $(x, y) \in C F R_{A}$. 
Please note that $O F R$ contains a triple for each pair of object flows sharing an object and $O b j$ is not allowed to contain objects not involved in object flow.

Definition 11 (refined activity diagram with object flow). $A$ refined activity diagram $R A_{O F}$ with object flow is a well-structured activity diagram $A_{O F}=(A, O b j, O F R, I$, $O)$ with coherent object flow such that each simple activity $x$ occurring in $A_{O F}$ is refined by a graph transformation rule $p_{x}$. Each decision activity $x \in A_{O F}$ has an if- or loop-guard which is equipped with a guard pattern $g$. Its guard rule $p_{g}$ is also denoted by $p_{x}$. Let $O_{p_{x}}$ be the output parameter set of $p_{x}$ and $I_{p_{y}}$ the input parameter set of $p_{y}$. OFR has to be coherent with refining rules which is defined as follows:

- For all $(x, o, y) \in O F R$ where $x \neq$ null, an output object parameter exists in $O_{p_{x}}$ which is called $\operatorname{src}(x, o, y)$. If $y \neq n u l l$, an input object parameter exists in $I_{p_{y}}$, called tgt $(x, o, y)$.

- For all triples $(x, o, y),\left(x, o, y^{\prime}\right)$ (resp. $\left.(x, o, y),\left(x^{\prime}, o, y\right)\right)$ in OFR we have $\operatorname{src}(x, o, y)=\operatorname{src}\left(x, o, y^{\prime}\right)\left(\operatorname{resp} \cdot \operatorname{tgt}(x, o, y)=\operatorname{tgt}\left(x^{\prime}, o, y\right)\right)$.

- For each two activities $x$ and $y$ and the set of all $(x, o, y) \in O F R$, the set of all pairs $(\operatorname{src}(x, o, y), \operatorname{tgt}(x, o, y))$ defines an injective mapping.

- For all triples $(x, o, n u l l),\left(x, o^{\prime}\right.$, null $)$ (resp. (null, o,y), (null, $\left.\left.o^{\prime}, y\right)\right)$ in OFR with $o \neq o^{\prime}$ we have $\operatorname{src}(x, o$, null $) \neq \operatorname{src}\left(x, o^{\prime}\right.$, null $)($ resp. $\operatorname{tgt}($ null $, o, y) \neq$ $\operatorname{tgt}\left(\right.$ null, $\left.\left.o^{\prime}, y\right)\right)$.

Definition 12 (semantics of refined activity diagrams with object flow). The semantics $\operatorname{Sem}\left(R A_{O F}\right)$ of an activity diagram $R A_{O F}$ with object flow being a refined activity diagram of $A_{O F}=(A, O b j, O F R, I, O)$ is equal to $\operatorname{Sem}(R A)$, the semantics of refined activity diagram $R A$ without object flow, where in addition partial rule dependencies (see Def. 1) are defined as follows:

For each pair of rules $\left(p_{i}, p_{j}\right)$ in a rule sequence $s: p_{1}, \ldots, p_{n}$ of $\operatorname{Sem}(R A)$ with $1 \leq i<j \leq n$, partial rule dependency $d_{i j}$ is defined as follows: Let $x$ (resp. $y$ ) be the activity that is refined by rule $p_{i}$ (resp. $p_{j}$ ) in sequence $s$, then the partial rule dependency $d_{i j}$ between $p_{i}$ and $p_{j}$ consists of all pairs $(\operatorname{src}(x, o, y), \operatorname{tgt}(x, o, y))$ such that $(x, o, y) \in O F R$ where src and tgt are given by Def. 11 .

$R A_{O F}$ is called dependency-compatible, if all rule sequences in $\operatorname{Sem}\left(R A_{O F}\right)$ are dependency-compatible, as defined in Def. 2 .

Definition 13 (completeness of refined activity diagrams with object flow). A set $\mathcal{S}$ of graphs is complete wrt. a dependency-compatible refined activity diagram $R A_{O F}$, if for all dependency-compatible rule sequences $s$ in $R A_{O F}$ there is a graph $G$ in $\mathcal{S}$ such that $s$ is applicable to $G$ in the sense of Def. 2

Properties quasi-completeness and consistency of refined activity diagrams without object flow can be extended to those with object flow accordingly.

Example 3 (Semantics of activity diagrams). The semantics of the flattened activity diagram AddLecture in Figure 2 consists of a number of rule sequences. For listing some of them, we use the following acronyms: NN=NotNull, CLec=CreateLecture, $\mathrm{CLab}=$ CreateLaboratory, $\mathrm{CEx}=$ CreateExercise, and SR=SetRoom: $\operatorname{Sem}\left(R A_{O F}\right) \supseteq$ $\{(C \operatorname{Lec}, \overline{N N}, \overline{N N}, \overline{N N}),(C \operatorname{Lec}, N N, S R, \overline{N N}, \overline{N N}),(C \operatorname{Lec}, \overline{N N}, N N, C \operatorname{Lab}, \overline{N N}, \overline{N N})$, 
$(C \operatorname{Lec}, \overline{N N}, N N, C L a b, N N, S R, \overline{N N}),(C \operatorname{Lec}, N N, S R, N N, C L a b, \overline{N N}, \overline{N N})$,

$(C L e c, \overline{N N}, N N, C L a b, N N, S R, N N, C E x, N N, S R)$,

$(C L e c, N N, S R, N N, C L a b, N N, S R, N N, C E x, N N, S R)\}$

As partly shown in Example 2, the object flow in our example can be formalized by partial rule dependencies. All rule sequences given above are dependency-compatible.

\section{Related Work}

This paper is rooted in formal semantics and analysis of activity diagrams as well as graph transformation approaches. While a lot of research has been done on semantics and validation of activity diagrams (see e.g. [11|12 9]), few works exist on the analysis of object flow in activity diagrams such as [13] and [14]. For example, [14] adds data flow semantics to activity diagrams by means of colored petri nets. Objects which are passed between activities have attribute value checks and method calls. Colored Petri nets provide validation like reachability of certain states and quantitative analyses as matching of time bounds. In contrast, we define a semantics for activity diagrams with object flow where activities may be refined by interrelated object diagrams which has not been done before (to the best of our knowledge).

Fujaba [15], VMTS [16], and GReAT [17] are graph transformation tools for specifying and applying object rules along a control flow specified by activity diagrams. Fujaba's story diagrams integrate activity diagrams with object rules. Compared to our approach, object flow is not depicted separately, but represented by equal names in activities. Furthermore, rules are not separated from activities. Rules used at different places have to be specified several times. We define object rules independently of activities and can apply them more than once with different arguments. VMTS and GReAT support controlled rule application with explicit control flow in a similar way and some kind of object flow. All three approaches are implemented, but do not provide a formal semantics comprising activity refinement and object flow.

\section{Conclusion}

In this paper, we have defined refined activity diagrams with object flow where each activity is refined by a set of interrelated object diagrams in addition, describing the pre- and post-conditions of an activity. Pre-conditions can also include non-existence conditions on object patterns. We have formalized the semantics of well-structured refined activity diagrams with coherent object flow using algebraic graph transformation where activity-refining object diagrams are defined by transformation rules. In addition, we have introduced the notion of partial dependencies between rules formalizing object flow between refined activities. To prepare a notion of consistency we define the applicability of rule sequences with partial rule dependencies.

In this paper, we have applied the approach to service modeling. Our example demonstrates how service behavior can be modeled precisely and how the coherence of its object flow can be checked. We expect that domains such as work flow design and aspect-oriented modeling can benefit from the application of our concepts as well. In future, we want to use the formal semantics given by graph transformation to prove the 
consistency of refined activity diagrams with object flow along sufficient criteria easy to check. We expect that the graph transformation environment AGG can do a good job to support automatic checks.

\section{References}

1. Lambers, L., Jurack, S., Mehner, K., Taentzer, G.: Sufficient Criteria for Consistent Behavior Modeling with Refined Activity Diagrams. In: Czarnecki, K., Ober, I., Bruel, J.-M., Uhl, A., Völter, M. (eds.) MODELS 2008. LNCS, vol. 5301, pp. 341-355. Springer, Heidelberg (2008)

2. Hausmann, J., Heckel, R., Taentzer, G.: Detection of Conflicting Functional Requirements in a Use Case-Driven Approach. In: Proc. of Int. Conference on Software Engineering 2002, Orlando, USA (2002)

3. Mehner, K., Monga, M., Taentzer, G.: Interaction Analysis in Aspect-Oriented Models. In: International Conference on Requirements Engineering RE 2006 (2006)

4. Lambers, L., Mariani, L., Ehrig, H., Pezzè, M.: A formal Framework for Developing Adaptable Service-Based Applications. In: Fiadeiro, J.L., Inverardi, P. (eds.) FASE 2008. LNCS, vol. 4961, pp. 392-406. Springer, Heidelberg (2008)

5. UML: Unified Modeling Language (2008), http: / / www . uml . org

6. Ehrig, H., Ehrig, K., Prange, U., Taentzer, G.: Fundamentals of Algebraic Graph Transformation. Springer, Heidelberg (2006)

7. Plump, D.: Evaluation of Funtional Expressions by Hypergraph Rewriting. PhD thesis, Universität Bremen, Fachbereich Mathematik und Informatik (1993)

8. AGG: AGG Homepage, http://tfs.cs.tu-berlin.de/agg

9. Engels, G., Soltenborn, C., Wehrheim, H.: Analysis of UML Activities Using Dynamic Meta Modeling. In: Bonsangue, M.M., Johnsen, E.B. (eds.) FMOODS 2007. LNCS, vol. 4468, pp. 76-90. Springer, Heidelberg (2007)

10. Jurack, S., Lambers, L., Mehner, K., Taentzer, G., Wierse, G.: Object Flow Definition for Refined Activity Diagrams - Long Verison. Technical Report 2009-1, Technische Universität Berlin (2009)

11. Eshuis, R., Wieringa, R.: Tool support for Verifying UML Activity Diagrams. IEEE Trans. on Software Eng. 7(30) (2004)

12. Störrle, H., Hausmann, J.H.: Towards a Formal Semantics of UML 2.0 Activities. In: Software Engineering 2005. LNI P-64, Gesellschaft f. Informatik, pp. 117-128 (2005)

13. Barros, J.P., Gomes, L.: Actions as Activities and Activities as Petri nets. In: Workshop on Critical Systems Development with UML. In: 20-24 workshop at 6. Int. Conf. on the Unified Modeling Language (UML 2003), San Francisco, U.S.A (2003)

14. Störrle, H.: Semantics and Verification of Data Flow in UML 2.0 Activities. Electronic Notes in Theoretical Computer Science, vol. 117 (2003)

15. Fischer, T., Niere, J., Torunski, L., Zündorf, A.: Story Diagrams: A new Graph Rewrite Language based on the Unified Modeling Language. In: Ehrig, H., Engels, G., Kreowski, H.J., Rozenberg, G. (eds.) TAGT 1998. LNCS, vol. 1764, pp. 296-309. Springer, Heidelberg (2000)

16. Visual Modeling and Transformation System (2008), http: / /vmts . aut . bme.hu/

17. GReAT - Graph Rewriting and Transformation (2008), http: / /www.isis.vanderbilt.edu/tools/GReAT 